\title{
INDICADORES URBANOS, BIOGRAMA, E ÍNDICES DE DESARROLLO SOSTENIBLE DEL HÁBITAT URBANO DEL VALLE DEL MANTARO PERIODO 1981-2017*
}

\author{
Martínez Vítor, César Fortunato ${ }^{1}$ \\ Facultad de Arquitectura de la Universidad Nacional del Centro del Perú
}

\begin{abstract}
RESUMEN
El estudio logra el objetivo de formular los indicadores urbanos, biograma e índices de desarrollo sostenible para que contribuyan a una sostenibilidad del hábitat urbano del valle del Mantaro. Se aplicó la metodología para estimar el nivel de desarrollo sostenible de los territorios rurales (Biograma) versión 2005 elaborado por el Instituto Interamericano de Cooperación para la Agricultura (IICA), y adaptado al medio urbano, en base a los Indicadores Urbanos Estambul+5, basado en la Agenda Hábitat y en las Resoluciones 15/6 y 17/1 de la Comisión de las Naciones Unidas para Asentamientos Humanos, logrando con ello obtener los resultados sobre la situación actual de sostenibilidad del hábitat urbano del valle del Mantaro, y sus proyecciones. Se aplica a las cuatro ciudades principales, capitales de provincia del valle del Mantaro y estas son; Huancayo metropolitano con sus distritos de El Tambo y Chilca, Jauja, Concepción y Chupaca. Los resultados muestran la utilidad práctica los indicadores urbanos, biograma e índices de desarrollo sostenible demostrando con ello la situación actual y previsible del hábitat urbano como un sistema inestable, con un crecimiento vegetativo de manera inercial, denotándose en términos generales un crecimiento espontáneo, conducentes a acrecentar la insostenibilidad a mediano plazo.
\end{abstract}

Palabras Clave: desarrollo sostenible urbano, indicadores urbanos.

\section{URBAN INDICATORS, BIOGRAMA AND INDEX OF SUSTAINED DEVELOPMENT OF THE URBAN HABITAT FROM THE MANTARO VALLEY PERIOD 1981-2017}

\begin{abstract}
The followsing study achieves the objective of formulating urban indicators, biograma and indexes of sustained development so that they contribute to a sustainability in the urban habitat of the Mantaro valley of he this study it was used the methodology to stimulate the level of sustainability in the rural areas (Biogram) versión 2005 elaborated by Interamerican Institute of Cooperation for the Agriculture (IICA), and adapted to the urban habitat of the Mantaro Valley he this study it was used the methodology to stimulate the level of sustituobiliti in the rural areas (Biogram) version 2005 elaborated by interamericam institute of cooperation for the agriculture (IICA) and adopted to the urban area based en the indicadores urban stambel +5 based in the habitat calendar and in the resolutions 15/6 and 17/1 of the commission of the United Nations for Human settlings achieving with it the results of current situations of sustamobility of the urban habitat in the Mantaro Valley and the in projections. It is applied to the four maincitios, capitals of provisue in the Mantaro Valley a not those are the center of Huancayo and its districts. The Tambo, Chilca, Jauja, Concepción and Chupaca. The results show that practical utility of urban indicators, biogram and indexes of sustained development demonstrating with it the present situation and presents the urban habitat, as an unstable system, with a vegetative growth in an inertial way, being denoted in general terms a spontaneous growth, directed to increase the insatiability to an intermediate term.
\end{abstract}

Key words: develop sustainable urban, urban indicators.

\footnotetext{
* Este trabajo de investigación fue recibido el 20/03/2008, retornado para su revisión el 22/09/2008 y aprobado para su publicación el 27/02/2009.
}

1. Email: cesar_vitor@hotmail.com 


\section{INTRODUCCIÓN}

Considerando que el indicador es una medida de la parte observable de un fenómeno que permite valorar otra porción no observable de dicho fenómeno, ésta debe permitirnos una lectura sucinta, comprensible y científicamente válida del fenómeno a estudiar que en nuestro caso es el Hábitat Urbano que permita resolver los diversos problemas existentes de información.

En efecto, más allá de la ocupación física de un territorio, el hábitat constituye el referente simbólico y social en el que se localiza el ser humano de una manera multidimensional. Así, el hábitat significa algo más que tener un techo bajo el cual protegerse: es, en definitiva, tanto el espacio físico como la forma social y personal de apreciarlo y apropiarlo.

En este sentido, lejos de ser algo homogéneo, simple y único, el hábitat cambia de un territorio - ciudad, región, nación - a otro, dependiendo de factores político - culturales, sociales, ambientales y económicos concretos. Desde esta perspectiva, no hay en la concepción del hábitat una "verdad objetiva" que refleje la realidad en su totalidad. Lo que encontramos son más bien aproximaciones que, construyéndola, son siempre inevitablemente parciales y están en permanente discusión

El crecimiento urbano genera una serie de procesos desestabilizadores, causa y efecto de insostenibilidad, que lleva una pérdida de calidad de vida, los cuales pueden agruparse en factores de: índole social; marginación, exclusión, pobreza, estratificación, descentralización, abandono de los centros históricos, efectos de la separación del entorno de trabajo y del hogar, perdida de la cultura propia, alienación, etc.

Índole económica; desempleo, terciarización excesiva de la economía, poca diversificación económica, dependencia de los recursos del exterior, movilidad creciente, necesidades energéticas de la economía local, etc.

Índole territorial y urbanística; calles para vehículos, escasez de espacios abiertos, edificios enfermos, pocas zonas de recreo, consumo de suelo desordenado en la perimétrica urbana, suburbanización, necesidades de infraestructura de transporte importantes, etc.
Índole ambiental; ruido, polución, congestión, contaminación atmosférica, residuos sólidos urbanos, falta de verde urbano, sobre-explotación de acuíferos y reservas de agua, agotamiento recursos naturales, consumo energético excesivo, etc.

Existen muchas vías para medir el fenómeno del hábitat urbano, se opta por el enfoque de indicadores de sostenibilidad que los organismos internacionales vienen desarrollando en eventos mundiales y que desde la escuela de Chicago años treinta en el marco de ecología urbana se basó en indicadores urbanos y esta goza en la actualidad de soporte técnico mundial y de popularidad dada su utilidad directa para la toma de decisiones locales. Se ha seleccionado los asentamientos urbanos del Valle del Mantaro por estar considerado como uno de los valles interandinos más importantes del Perú, y las ciudades con más de 10 000 habitantes, entre los cuales están las capitales de las provincias de Huancayo, Jauja, Chupaca, y Concepción, porque pertenecen a la problemática local y microregional en la que estamos insertos. La vivimos y vemos permanentemente, como en un proceso relativamente corto se transforma irreversiblemente a su destrucción, por falta de estudios que midan este proceso y permita autorregularse a través del manejo de indicadores e instrumentos metodológicos que visualicen y modelasen los escenarios presentes y futuros que ayuden a superar las amenazas y riesgos para un desarrollo sostenible.

En el presente informe se ha logrado el objetivo general de la investigación, que es la de formular los indicadores urbanos, biograma e índices de desarrollo sostenible para que contribuyan a una sostenibilidad autorregulada del hábitat urbano del Valle del Mantaro. Se afirma que la formulación de los indicadores urbanos, biograma e índices de desarrollo sostenible contribuyen de manera directa a la gestión del desarrollo sostenible del hábitat urbano del Valle del Mantaro.

\section{MATERIAL Y MÉTODOS}

El primer documento de referencia esta constituida por los Indicadores Urbanos Estambul+5, basado en la Agenda Hábitat y en las Resoluciones $15 / 6$ y 17/1 de la Comisión de las Naciones Unidas para Asentamientos 
Humanos, la CNUAH (Hábitat) ha elaborado un sistema de indicadores que contiene un conjunto de 23 indicadores clave y nueve listas de datos cualitativos.

De este sistema de indicadores se ha podido procesar de manera análoga 11 indicadores distribuidos de la siguiente manera; dos del subsistema económico, cuatro del subsistema social, y cinco del subsistema ambiental. Los datos obtenidos para la formulación de los indicadores proceden en primera instancia de los censos nacionales de 1981, 1993 y 2005 proyectados al 2017, y de fuentes sectoriales, gobiernos locales, e instituciones especializadas como el CONAM y otros citados como fuentes en cada caso. El procedimiento seguido se describe de manera detallada para cada indicador, considerando los factores de confiabilidad de las fuentes de procedencia.

El segundo documento base está constituido por la metodología para estimar el nivel de desarrollo sostenible de los territorios rurales (Biograma) versión 2005 elaborado por el Instituto Interamericano de Cooperación para la Agricultura (IICA). Por sus características representa de manera didáctica el estado y la evolución del desarrollo sostenible de la unidad de análisis cuyo marco es el Valle del Mantaro y las cuatro ciudades principales tomadas como muestra, el software es bastante amigable y admitió y proceso los indicadores urbanos.

Cuadro 1. Resumen de cálculos de indicadores generales para el Valle del Mantaro.

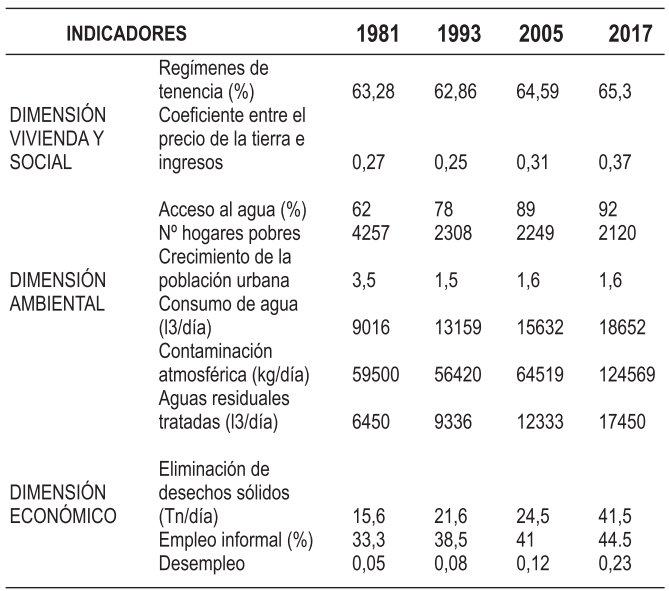

Fuente: elaboración propia.

\section{RESULTADOS}

Posterior a la selección de las unidades de análisis y los períodos de tiempo, se definieron tres dimensiones de análisis: la ambiental, la económica y la social. En otras palabras, se definió una forma funcional del desarrollo sostenible, la cual tenía como variables explicativas la dimensión ambiental (A), económica (E) y social (S), los cuales fueron ponderados de acuerdo al siguiente gráfico.

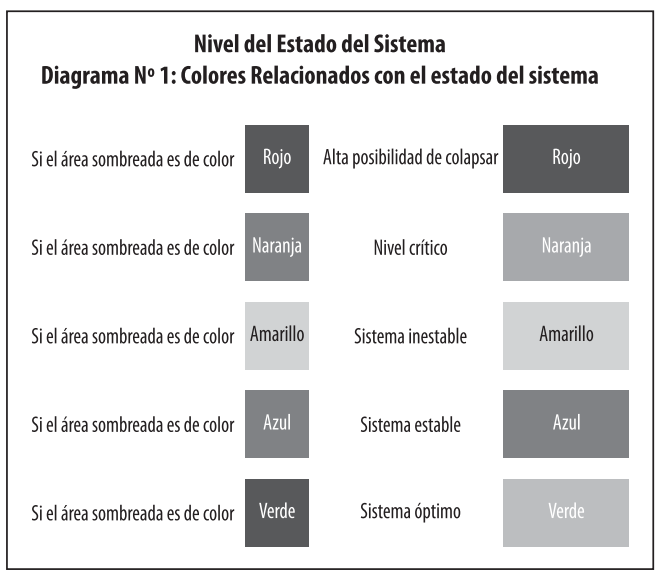

Fuente: Valle del Mantaro.

Presenta desde el año 1981 a la proyección 2017 el ponderado delascuatro ciudades con suscaracterísticas propias que por la extensión del presente documento no se comenta de manera individualizada sino como un resumen general.

En la dimensión vivienda y social valores crecientes positivos el régimen de tenencia de $63 \%$ a un $65.3 \%$, acceso al agua de $62 \%$ a un $92 \%$, valores decrecientes positivos el número de hogares pobres de 4257 a un 2120 y valores crecientes negativos el coeficiente entre el precio de la tierra e ingresos de 0,27 a un 0,37.

En la dimensión ambiental valores decrecientes positivos el crecimiento de la población de 3,5 a un 1,6, valores crecientes negativos el consumo del agua de 9016 /3/día a un 18652 I3/día, contaminación atmosférica de $59500 \mathrm{~kg} / \mathrm{día}$ a $124569 \mathrm{~kg} / \mathrm{día}$, aguas residuales tratadas de 6450 a 17450 y eliminación de desechos sólidos de 15,6 tn/día a 41,5 tn/día, presentándose los tres últimos como indicadores críticos. 
En la dimensión económica valores crecientes negativos el empleo informal de $33,3 \%$ a un $44,5 \%$, y el índice de desempleo de 0,05 a 0,23 presentándose como indicadores críticos.

De una visualización en cifras para el caso del Valle del Mantaro, es recurrente la situación que se presenta en cada una de las ciudades analizadas, todos los indicadores sean estos positivos o negativos es de manera ascendente, que indica un crecimiento vegetativo de manera inercial, denotándose en términos generales un crecimiento espontáneo. Teniendo como cifras representativas de los once indicadores tres con valores positivos y ocho con valores negativos para el desarrollo sostenible.

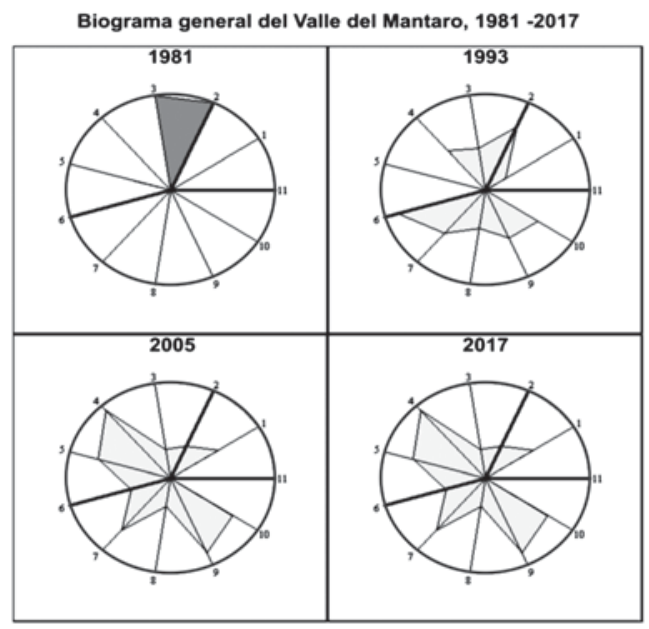

El gráfico muestra para 1981 un nivel crítico que se ha ido superando a una situación inestable para el 2005 y se proyecta de seguir el curso de la situación actual a mantenerse inestable, esta situación debe revertirse con políticas que ataquen los ocho indicadores negativos que se detectaron en el estudio, a fin de propender a una situación de un sistema estable.

\section{Logros de la investigación}

1. En el desarrollo de la investigación se ha logrado formular los indicadores urbanos, biograma e índices de desarrollo sostenible para que contribuyan a una sostenibilidad del hábitat urbano del Valle del Mantaro. Su aplicación estará sujeta a los actores de la aplicación de políticas de desarrollo.
2. Se han formulado los indicadores urbanos, biograma e índices de desarrollo sostenible de la dimensión social, económica, y ambiental del hábitat urbano para en el proceso de planeamiento sostenible del hábitat urbano del valle del Mantaro puedan tomarse decisiones jerarquizadas por cada dimensión.

3. De los resultados se ha identificado en términos generales en la seis ciudades incluyendo la capital de la región, presentan características análogas, con un crecimiento vegetativo de manera inercial, denotándose en términos generales un crecimiento espontáneo, conducentes a acrecentar la insostenibilidad a mediano plazo.

4. El sistema de generar indicadores urbanos, biograma e índices de desarrollo sostenible de la dimensión social, económica, y ambiental del hábitat urbano, es de una necesidad imprescindible y urgente para que se pueda medir de manera permanente nuestra opción de desarrollo, y no solamente un crecimiento anárquico, como se presenta en la actualidad y sus proyecciones.

\section{DISCUSIÓN}

La estimación del desarrollo sostenible de territorios rurales y urbanos, por medio del biograma, es un primer paso en el proceso de instrumentar de procesos racionales y analíticos en la medición del desarrollo sostenible.

Desde Río 92, punto de partida de la propuesta conocida como agenda 21 , donde se plantea entre las múltiples necesidades la de poder planear, ejecutar, medir, controlar y evaluar las gestiones tendientes a fomentar el desarrollo sostenible. Como se demuestra en la presente investigación cuán difícil es formular los indicadores debido a la escasa información sistematizada, y su utilidad de suma importancia para la toma de decisiones en cualquier tipo o modalidad de gestión.

En nuestro país se adolece de organismos que asuman este reto, como se puede evidenciar el tema de ordenamiento territorial forma parte de un cuarto escalón en las oficinas del ministerio de Vivienda con escaso personal reducido a su mínima 
expresión, dejando pendiente esta tarea, que a la postre se convierte en el primer escollo a superar cuando se trata de temas de ordenamiento territorial, acondicionamiento territorial, y ordenamiento urbano.

\section{LITERATURA CITADA}

Castro, M., 2002. Indicadores de desarrollo sostenible urbano. Una aplicación para Andalucía. Tesis doctoral, Universidad de Málaga. 540-23.

CNUAH - PGU - ALC: 2001. Indicadores Urbanos Estambul +5 , Guía y ficha de encuesta. PGU -ALC Quito-Ecuador. 54-3.

CNUMAD, 1992. Informe de la conferencia de las Naciones Unidas sobre el medio ambiente y el desarrollo. Naciones Unidas, Nueva York. 468456.

Constitución Política del Perú. 1993. Congreso Constituyente Democrático.

Enkerlin, E; Cano, G; Garza, R; y Vogel, E., 1995. Ciencia ambiental y desarrollo sostenible. México DF.; 690 - XV.

Gallopin, G., 2006. Los Indicadores de desarrollo sostenible:aspectosconceptualesmetodológicos. Ponencia realizada para el seminario de expertos sobre indicadores de sostenibilidad en la formulación y seguimiento de políticas. Proyecto FODEPAL. Santiago de Chile. 36-7.

Giraldo, F.; García, J.; Bateman, A. y Alonso, A., 2006. Hábitat y pobreza, los objetivos de desarrollo del milenio desde la ciudad. ONU-Hábitat. JavegrafBogota. 180 -24.
Ministerio de Vivienda y Construcción. 2003. Reglamento de acondicionamiento territorial y desarrollo urbano. Lima: Decreto Supremo № 027-2003-Vivienda del 03 de octubre de 2003.

Ministerio de Vivienda, Construcción y Saneamiento. 2004. Resolución Ministerial № 021-2004Vivienda, reglamento de organización y funciones del Ministerio de Vivienda, Construcción y Saneamiento.

Ministerio de Vivienda. 1975. Plan nacional de desarrollo urbano 1975 - 1990. Lima: R. S. № 144-75-VC-1100 de fecha 11 de junio de 1975.

Programa de trabajo propuesto del Programa de las Naciones Unidas para los Asentamientos Humanos (hsp / gc /19/8), para el Bienio 2004 - 2005 (http://www.unhabitat.org/ governingbodies/pre_session_documents.asp).

Propuesta lineamientos de política para el ordenamiento territorial, Documento aprobado por el Subcomité de OT el 1. Nov. 05 documento aprobado por el Petit Comité de OT el $12 \mathrm{Jul}$. 06 documento aprobado por el Comité Técnico Consultivo 20 Jul. 06.

Sepúlveda, S.; Chavarría, H. y Rojas, P., 2005. Metodología para estimar el nivel de desarrollo sostenible de los territorios rurales (El Biograma). IICA. 67-7. 